\title{
Electromagnetically Induced Guiding and Superradiant Amplification of Counter-Propagating Lasers in Plasma
}

\author{
G. Shvets and N. J. Fisçh \\ Princeton Plasma Physics Laboratory, Princeton University, Princeton, NJ 08543
}

A. Pukhov

Max-Plank-Institut fur for Quantenoptik, D-85748 Garching, Germany

\begin{abstract}
The interaction of counter-propagating laser pulses in a plasma is considered. When the frequencies of the two lasers are close, nonlinear modification of the refraction index results in the mutual focusing of the two beams. A short (of order the plasma period) laser pulse can be nonlinearly focused by a long counter-propagating beam which extends over the entire guiding length. It is also demonstrated that a short $\left(<1 / \omega_{p}\right)$ laser pulse can be superradiantly amplified by a counter-propagating long low-intensity pump while remaining ultra-short. Particle-in-Cell simulations indicate that pump depletion can be as high as $40 \%$. This implies that the long pump is efficiently compressed in time without frequency chirping and pulse stretching, making the superradiant amplification an interesting alternative to the conventional method of producing ultra-intense pulses by the chirped-pulse amplification.
\end{abstract}

\section{INTRODUCTION}

One of the important challenges to laser-plasma accelerators is to propagate an intense laser pulses over a long distance. A laser will, in free space, remain focused over a Rayleigh range $Z_{R}=\pi \sigma^{2} / \lambda_{0}$, where $\lambda_{0}$ is the laser wavelength, and $\sigma$ is the laser spot size at the focus. Relativistic self-focusing, which can overcome diffraction, is ineffective for short laser pulses and requires a very high laser power $P_{c 1}=17 \omega_{0}^{2} / \omega_{p}^{2} \mathrm{GW}$, where $\omega_{p}^{2}=4 \pi n_{0} e^{2} / m$ is the plasma frequency, $n_{0}$ is the background electron density, and $-e$ and $m$ are the electron density and mass, respectively.

In this paper we demonstrate how a second counter-propagating pulse can be used to create an "electromagnetic channel". Such electromagnetically induced guiding (EIG) occurs when the intensity of the guiding laser is well below the relativistic threshold $P_{c 1}$. The guiding and guided beams interfere, forming a periodic intensity 


\section{DISCLAIMER}

This report was prepared as an account of work sponsored by an agency of the United States Government. Neither the United States Government nor any agency thereof, nor any of their employees, make any warranty, express or implied, or assumes any legal liability or responsibility for the accuracy, completeness, or usefulness of any information, apparatus, product, or process disclosed, or represents that its use would not infringe privately owned rights. Reference herein to any specific commercial product, process, or service by trade name, trademark, manufacturer, or otherwise does not necessarily constitute or imply its endorsement, recommendation, or favoring by the United States Government or any agency thereof. The views and opinions of authors expressed herein do not necessarily state or reflect those of the United States Government or any agency thereof. 


\section{DISCLAIMER}

Portions of this document may be illegible in electronic image products. Images are produced from the best available original document. 
pattern which ponderomotively drives the plasma wave. The guided beam undergoes a Bragg reflection off this periodic density perturbation, scattering into the guided pulse. Basic equations for the plasma wave excitation and laser scattering are given in Section II

When laser intensities are small, plasma wave remains linear, and the complex index of mutual refraction $\chi_{0}$ can be introduced. Depending on the frequency detuning between the lasers, duration of the guided pulse, and the transverse intensity profile of the guiding beam, the real part of $\chi_{0}$ can have a maximum on axis, resulting in the focusing of the pulse. It turns out that the most power-efficient way of guiding a short (of order plasma period) pulse is to employ a long (twice the desired propagation distance) low-intensity Bessel beam. The theory and numerical simulations of the EIG of long and short pulses is presented in Section III.

The imaginary part of the mutual refraction index describes the energy exchange between the two beams. If the frequency detuning between the beams is close to $\omega_{p}$, the lower-frequency (signal) beam can be amplified at the expense of the higherfrequency (pumping) beam. This linear amplification has an important limitation: pulses shorter than a plasma period broaden because of the narrow bandwidth of the Raman backscattering instability. Building up on our earlier work [1] on superradiance in Raman backscattering, we found an alternative technique for nonlinearly amplifying ultra-short laser pulses, which is described in Section IV.

\section{BASIC EQUATIONS}

Consider two circularly polarized counter-propagating laser pulses $\vec{a}_{0}$ and $\vec{a}_{1}$, where $\vec{a}_{0,1}=a_{0,1} / 2\left(\vec{e}_{x} \pm i \vec{e}_{y}\right) e^{i \theta_{0,1}}+$ c.c. are the normalized vector potentials: $a_{0,1}\left(\vec{r}_{\perp}, z, t\right)=e A_{0,1} / m c^{2}$. Depending on the problem at hand (guiding or amplification), the subscripts 0 and 1 distinguish the right-moving guided (or amplified) pulse and the left-moving guiding (or pumping) pulses, respectively. The phases of the waves are $\theta_{0}=\left(k_{0} z-\omega_{0} t\right)$ and $\theta_{1}=\left(k_{1} z+\omega_{1} t\right)$, and we choose $|\Delta \omega|=\left|\omega_{0}-\omega_{1}\right| \ll \omega_{0}$. We also assume a tenuous plasma $\omega_{p} \ll \omega_{0,1}$, so that $k_{0} \approx k_{1} \approx \omega_{0} / c$.

Assuming that both lasers are of nonrelativistic intensity, $a_{0,1} \ll 1$, the dominant nonlinear force experienced by the plasma electrons is the ponderomotive $\vec{v}_{1} \times \vec{B}_{0}+$ $\vec{v}_{0} \times \vec{B}_{1}$ force, where $\vec{v}_{0,1}=c \vec{a}_{0,1}$. On a time-scale much longer than $1 / \omega_{0}$ plasma electrons are pushed by the intensity gradient of the "optical lattice" which is produced by the interference of the two beams and has a spatial periodicity $k_{0}+k_{1} \approx$ $2 k_{0}$. Therefore, the nonlinear force experienced by an electron $j$ is a function of its position in the optical lattice, which is characterized by its ponderomotive phase $\psi_{j}=\left(k_{0}+k_{1}\right) z_{j}-\Delta \omega t_{j}$. As was shown earlier [1], the equations of motion take the form

$$
\ddot{\psi}_{j}+\omega_{B}^{2} \sin \psi_{j}=-2 \omega_{p}^{2} \sum_{l=1}^{\infty} \frac{\cos \psi_{j}<\cos \psi_{j}>+\sin \psi_{j}<\sin \psi_{j}>}{l}-\frac{2 \omega_{0} e E_{z}}{m c^{2}}
$$


where $\omega_{B}^{2}=4 \omega_{0}^{2} a_{0} a_{1}$ is the bounce frequency of an electron in the optical lattice, and averaging is over one lattice period.

The second term in the LHS of Eq. (1) is the ponderomotive force, the sum in the RHS is over the harmonics of the rapidly spatially varying space-charge force, and $E_{z}$ is the global electric field. The average of $E_{z}$ over the lattice period does not vanish. The origin of $E_{z}$ was explained in Ref. [1]: as the photons are exchanged between the counter-propagating beams, electrons receive the recoil momentum. However, the average current in 1-D is equal to zero - electrons cannot run away from the immobile ions. To ensure this an electric field $E_{z}$ is produced, satisfying $\partial E_{z} / \partial t=4 \pi e n_{0}<v_{z j}>$.

The periodic density variation, induced in the plasma, serves as an index grating which causes the $a_{1}$ to undergo Bragg backscattering into $a_{0}$, and vice versa. The nonlinear interaction between the beams is calculated by substituting the modified plasma density into the respective paraxial wave equations. For example, for the guided pulse obtain $2 i k_{0}\left(\frac{\partial}{\partial z}+\frac{1}{c} \frac{\partial}{\partial t}\right) a_{0}+\nabla_{\perp}^{2} a_{0}=-k_{p}^{2} \chi_{0} a_{0}$, where $\chi_{0}=-\left\langle e^{-\psi_{j}}>a_{1}^{*} / a_{0}\right.$ is the mutual refraction index witnessed by the beam 0 .

Refraction index can be evaluated in closed forms if plasma motion is linear, which is the case if $\omega_{B}^{2} \ll \omega_{p}^{2}$ :

$$
\chi_{0}(\tau)=\frac{2 \omega_{0}^{2}\left|a_{1}\right|^{2}}{\omega_{p}} \int_{0}^{\infty} d u \sin \left(\omega_{p} u\right) e^{i \Delta \omega u} \frac{a_{0}(\tau-u)}{a_{0}(\tau)}
$$

where $\tau=t-z / c$ is a co-moving with the pulse 0 coordinate, and the guiding (pumping) pulse is assumed very long. As apparent from Eq. (2), refraction index scales as $\chi_{0} \propto|a|^{2} \omega_{0}^{2} / \omega_{p}^{2}$. For co-propagating beams (or a single relativistic beam) this scaling would change to $\chi_{R} \propto|a|^{2}$. The enhancement factor $\omega_{0}^{2} / \omega_{p}^{2}$ is due to the fact that the ponderomotive force is proportional to the gradient of the laser intensity, which is the largest for the small-scale $\approx \lambda_{0} / 2$ interference pattern of the counter-propagating beams.

\section{LINEAR THEORY OF EIG}

The mutual refraction index $\chi_{0}$ is, in general, complex. The real part of $\chi_{0}$ is much larger than the imaginary part when the beams are detuned far from the plasma resonance, $\left|\Delta \omega^{2}-\omega_{p}^{2}\right| \geq \omega_{p}^{2}$. For such detunings there is no energy exchange between beams, and their interaction is purely refractive. This is assumed for the rest of this section. The nonlinear interaction between $a_{0}$ and $a_{1}$ can then lead to electromagnetically-induced guiding (of one or both beams) if $\chi_{0}$ is peaked on axis. The refraction index depends on the spatial and temporal profiles of the pulses and their frequency detuning. For long pulses $\tau_{L} \gg \min \left(1 / \Delta \omega, 1 / \omega_{p}\right)$ the mutual refraction index is independent of $\tau: \chi_{0}=2\left|a_{1}\right|^{2} \omega_{0}^{2} /\left(\omega_{p}^{2}-\Delta \omega^{2}\right)$. Thus, two long transversely Gaussian beams focus each other if $\Delta \omega<\omega_{p}$. Since this type 
of nonlinear guiding relies on a rather delicate mechanism of generating an index grating with a very short wavelength, and then backscattering off this grating, direct Particle in Cell (PIC) simulation in a slab geometry are used to demonstrate the existence of the EIG.

A 2D version of the relativistic electromagnetic PIC code VLPL (Virtual Laser Plasma Lab) [2] running on a single processor workstation was used to simulate the problem. The grid size was $4000 \times 120$ with $4 \times 10^{6}$ electrons on it. The two counter-propagating laser pulses with wavelengths $\lambda_{1}=\lambda_{0}=1 \mu \mathrm{m}$ are focused to the spotsizes $\sigma_{0}=\sigma_{1}=3 \mu \mathrm{m}$ at their corresponding entrances into a $400 \mu \mathrm{m} \times 40 \mu$ slab of $n_{0}=10^{19} \mathrm{~cm}^{-3}$ plasma. The normalized vector potentials of both lasers at their respective foci are equal to $\alpha_{0}=0.07$. The intensity gray scale plots for the right-moving pulse in vacuo and in the plasma are shown in Figs. 1(b) and (c), respectively. The snapshots at $t=500 \lambda_{0} / c$ of the on-axis $(x=0)$ laser intensities for both cases are plotted versus the propagation distance in Fig. 1(a). As Fig. 1(a) demonstrates, in the presence of the plasma the intensity of the right-going laser $\left|a_{0}\right|^{2}\left(z=L_{g}\right)$ is increased by a factor 2 . Thus, the nonlinear interaction between the lasers strongly reduces laser spreading, confirming the electromagnetically induced guiding. Note that in this example EIG occurs at a very modest laser power $P / P_{\mathrm{c} 1}=0.0016$.

A simplified analytical treatment of the EIG can be developed by assuming that the guided laser pulse has a Gaussian transverse profile when it enters the plasma, and that it remains such in the plasma. Guicied beam is assumed to have an intensity profile $|a|_{0}^{2}=\left(1 / R_{0}^{2}\right) \alpha_{0}^{2} \exp \left[-r^{2} / R_{0}^{2} \sigma^{2}\right]$ in cylindrical geometry, where $R_{0}$ is the dimensionless spotsize. Applying the source-dependent expansion to the envelope equations for $a_{0}$ similarly to the way it was done in Ref. [3], the equation

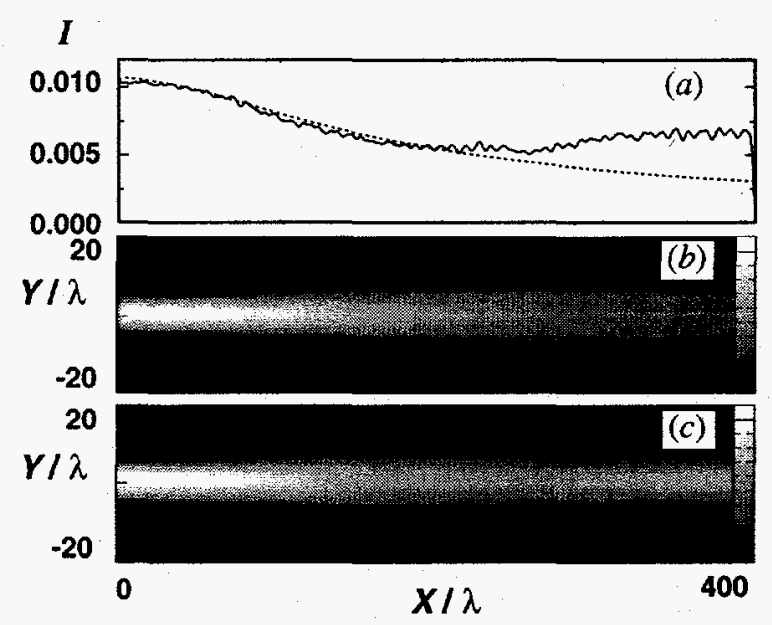

FIGURE 1. (a) On-axis intensity of a right-going laser focused at $z=0$ in vacuum (dashed line) and in the plasma (solid line). Contour plot of the intensity of the right-going laser in vacuum (b) and in the plasma (c). 
is derived for the normalized beam radius:

$$
\frac{d^{2} R_{0}}{d \bar{z}^{2}}=\frac{4}{R_{0}^{3}}+\frac{4 k_{p}^{2} \sigma^{2}}{R_{0}^{3}} \int_{0}^{+\infty} d \rho \rho^{2} e^{-\rho^{2} / R_{0}^{2}} \frac{\partial \chi_{0}}{\partial \rho}
$$

where $\rho=r / \sigma$, and $d / d \bar{z}=2 k_{0} \sigma^{2}(\partial / \partial z+\partial / c \partial t)$. In Ref. [4] a similar equation was derived for flat beams in order to make a comparison with the PIC simulation.

Guiding of ultra-short (of order $1 / \omega_{p}$ ) pulses is particularly interesting for laserwakefield acceleration since such pulses are optimal for generating plasma wakes. For short guided pulses $\chi_{0}$ becomes a function of $\tau$. This is because, as Eq. (2) indicates, $\chi_{0}(\tau)$ of a given longitudinal laser slice $\tau$ is determined by the laser intensity at all earlier instances $\tau^{\prime}<\tau$. Therefore, the nonlinear focusing experienced by the short pulse is not uniform along the pulse and determined by its longitudinal profile. One finds that, if the pulse intensity drops off faster than exponentially in $|\tau|$, the ratio $a_{0}(\tau-u) / a_{0}(\tau)$ decreases with $|\tau|$. For example, for a longitudinally Gaussian profile $a_{0}=\alpha_{0} \exp \left(-\tau^{2} / 2 \tau_{L}^{2}\right)$ the leading edge $\tau<-\tau_{L}$ slowly erodes because $\chi_{0}(\tau) \propto \tau_{L}^{2} / \tau^{2}$. However, for $a_{0}=\alpha_{0} \sec \left(\tau / \tau_{L}\right)$ (exponential decay in $|\tau|$ ) the leading edge is uniformly focused: $\chi_{0}=2\left|a_{1}\right|^{2} \omega_{0}^{2} \tau_{L}^{2} /\left(1+\omega_{p}^{2} \tau_{L}^{2}\right)$ for $\Delta \omega=0$. Note that guiding is reduced for ultra-short pulses because the plasma does not respond faster than on a $1 / \omega_{p}$ time scale.

The ultra-short pulse is guided by the long guiding pulse along its entire passage through the plasma. In contrast, the guiding pulse is only briefly affected by the guided pulse. Therefore, it has to propagate through the entire plasma region without any additional nonlinear focusing by the short pulse. We found that the most power-efficient choice of the guiding beam is a Bessel beam.

Apertured Bessel beams [5] with sharply peaked radial profiles propagate without diffraction over the distances much exceeding the Rayleigh length (as defined using the width of the central maximum for a spotsize). Such beams transport energy within a narrow spot $\sigma_{1} \ll W$ over the distance of order $L_{g}=2 \pi W \sigma_{1} / \lambda_{1}$, where the laser intensity profile is given by $\left|a_{1}\right|^{2}=\alpha_{1}^{2} J_{p}^{2}\left(r / \sigma_{1}\right)$, and the beam is apertured at the radius $W \gg \sigma_{1}[5]$. The total power of a Bessel beam is given by $P_{B p} / P_{0}=$ $\pi W \sigma_{1}\left|\alpha_{1}\right|^{2} / \lambda_{0}^{2}$, where $P_{0}=m c^{3} / r_{e}=8.0 \mathrm{GW}$ and $r_{e}=e^{2} / m c^{2}$ is the classical electron radius. Therefore, the propagation distance is proportional to the beam power.

A large spotsize Gaussian beam, such that its Rayleigh length is equal to the required guiding distance $L_{g}$, may appear to be an alternative to the Bessel beam. . At first glance, the on-axis mutual refraction index $\chi_{0}$, which is proportional to the peak intensity of the guiding beam, would be, roughly, the same for the equal power Gaussian and Bessel beams. This is because the product of the peak on-axis intensity and the propagation distance is, approximately, the same for the equal power Gaussian and Bessel beams [6]. However, following Eq. (3), the focusing strength of the guiding beam is proportional to the curvature of $\chi_{0}$, and is, therefore, larger for a Bessel beam.

The choice of $p$, the order of the Bessel beam, depends on the frequency detuning of the lasers. Consistently with our assumption of purely refractive interaction 
between beams, we consider two cases (i) zero detuning $\Delta \omega=0, p=0$, and (ii) $\Delta \omega \gg \omega_{p}, p=1$. For a zero-detuning case assume $a_{0}=\alpha_{0} \sec \left(\tau / \tau_{L}\right)$ to facilitate the calculation of $\chi_{0}$ at the leading edge: $\chi_{0}=2\left|a_{1}\right|^{2} \omega_{0}^{2} \tau_{L}^{2} /\left(1+\omega_{p}^{2} \tau_{L}^{2}\right)$. Clearly, $\chi_{0}$ peaks on axis for a zeroth order Bessel beam.

The guiding power threshold $P_{B 0}$ is estimated by substituting $\left|a_{1}\right|^{2}=\alpha_{1}^{2} J_{0}^{2}\left(r / \sigma_{1}\right)$ into Eq. (3) and assuming $R_{0}=1$ :

$$
k_{0}^{2} \sigma^{2}\left|\alpha_{1}\right|^{2} \frac{2 \omega_{p}^{2} \tau_{L}^{2}}{1+\omega_{p}^{2} \tau_{L}^{2}}\left[t \frac{d}{d t}\left(e^{-t} I_{0}(t)\right)\right]=-1,
$$

where $t=\sigma^{2} / 2 \sigma_{1}^{2}$ and $I_{0}(t)$ is a modified Bessel function. The strongest focusing occurs for $\sigma_{1} \approx 0.8 \sigma$. Since the guiding distance $L_{g}$ is related to the beam power $P_{B 0}$, Eq. (4) can be rewritten as a power threshold for the EIG:

$$
P_{B 0}=1.27 \mathrm{GW} \frac{1+\omega_{p}^{2} \tau_{L}^{2}}{\omega_{p}^{2} \tau_{L}^{2}} \frac{L_{g}}{k_{0} \sigma^{2}} .
$$

The EIG threshold $P_{B 0}$ differs from the relativistic guiding threshold $P_{c 1}$ in two respects: first, it is independent of the plasma density, enabling electromagneticallyinduced channeling in very tenuous plasmas, and, second, it depends on the propagation distance. In deriving Eq. (2) linear plasma response was assumed, which is only valid when $\left|\chi_{0}\right|<1$. Combining this restriction with the guiding condition, given by $\mathrm{Eq}$. (4), imposes an upper limit on the intensity of the guided pulse:



FIGURE 2. Superradiant amplification by factor 100 , results of the time-averaged code. Pump $a_{1}=0.025$, initial signal $a_{0}=0.025$ and $\tau_{L}=1 / \omega_{p}$, plasma $n_{0}=2.5 \times 10^{18} \mathrm{~cm}^{-3}$. 
$\alpha_{0} \leq \lambda_{0} \sigma / \lambda_{p}^{2}$. More extensive numerical study is needed to fully understand how guiding is affected when the linear theory breaks down, but it is reasonable to expect that guiding saturates and weakens at higher intensities of the guided pulse.

Since the focusing strength is determined by the curvature of the $\chi_{0}$ rather than by its absolute value, a possible solution to the nonlinear saturation of the EIG is to use a first-order Bessel beam, which has an intensity node on axis. The density perturbation on axis vanishes while its curvature does not. To utilize the $J_{1}$ Bessel beam frequency detuning has to be chosen $\Delta \omega>\omega_{p}, \tau_{L}^{-1}$. For a guided beam of arbitrary longitudinal shape $\chi_{0} \approx 2\left|a_{1}\right|^{2} \omega_{0}^{2} /\left(\omega_{p}^{2}-\Delta \omega^{2}\right)$, enabling focusing by a beam with intensity minimum on axis. The guiding condition similar to Eq. (4) can be derived for the $J_{1}\left(r / \sigma_{1}\right)$ beam. The strongest focusing occurs for $\sigma_{1}=1.04 \sigma$, yielding $k_{0}^{2} \sigma^{2} \alpha_{1}^{2}=5\left[\left(\Delta \omega / \omega_{p}\right)^{2}-1\right]$, from which the power threshold condition is be derived:

$$
P_{B 1}=3.6 \mathrm{GW}\left(\Delta \omega^{2} / \omega_{p}^{2}-1\right) \frac{L_{g}}{k_{0} \sigma^{2}} .
$$

The linear theory breaks down at $r \approx \sigma$ if $\alpha_{0}>2.7 \lambda_{0} \sigma / \lambda_{p}^{2}$. In practice, this limitation is likely to be over-stringent since the nonlinear saturation of the EIG in a limited spatial region may not strongly influence the overall focusing. As a numerical example, consider guiding a $\tau_{L}=10 \mathrm{fs}$ long, $\sigma=18 \mu \mathrm{m}$ wide laser pulse through $1 \mathrm{~cm}$ of $n_{0}=10^{19} \mathrm{~cm}^{-3}$ plasma. For $\Delta \omega / \omega_{p}=1.5$ the threshold power of the Bessel beam $P_{B_{1}} \approx 16 \mathrm{GW}$. This beam can guide a pulse with a normalized vector potential up to at least $\alpha_{0}=0.5$, or $2.2 \mathrm{TW}$.

\section{SUPERRADIANT AMPLIFICATION OF ULTRA-SHORT PULSES}

In this Section we consider the possibility of amplifying an ultra-short laser pulse by colliding it with a long counter-propagating pump. If the frequency of the short pulse $\omega_{0}$ is smaller than the pump frequency $\omega_{1}$, Manley-Rowe relations dictate the direction of the energy flow - from the pump to the pulse. The difficulty is to sustain the short duration of the signal $\tau_{L} \approx \omega_{p}^{-1}$ as its intensity grows by several orders of magnitude. In the linear (exponential) regime of amplification the signal inevitably broadens due to the narrow amplification bandwidth of the Raman backscattering (RBS). It turns out that in the nonlinear regime, defined by $\omega_{B}>\omega_{p}$, superradiant amplification (SRA) [1] is possible. In the course of the SRA the width of the signal's leading spike is about $\tau_{L} \approx \pi / \omega_{B}$. Since $\omega_{B} \propto \sqrt{a_{0}}$, the signal slowly narrows down as it is amplified.

We simulated a collision between a short $\tau_{L}=\omega_{p}^{-1}$ signal of initial amplitude $a_{0}=$ 0.025 and a long pump with $a_{1}=0.025$ using the one-dimensional time-averaged particle code, specifically developed for the study of the RBS instability [1]. This code solves the plasma equation of motion (1) and times-averaged (eikonal) wave equations for the forward and backwards radiation. Simulation results are presented 
in Fig. 2. Plasma density was chosen $n_{0}=2.5 \times 10^{18} \mathrm{~cm}^{-3}$, and the frequency detuning between the signal and the pump is $\Delta \omega=-1.7 \omega_{p}$. As shown in Fig. 2, the signal is amplified by a factor 100 in intensity to a mildly relativistic $I=2 \times 10^{17}$ $\mathrm{W} / \mathrm{cm}^{2}$. It's final FWHM is about one-third of the original.

The initial signal amplitude $a_{0}$ was chosen to ensure that $\omega_{B} \approx \omega_{p}$ at the entrance into the plasma, so that the nonlinear regime is accessed from the start. Taking a smaller initial $a_{0}$ was resulting in the initial spreading of the pulse. This is consistent with the earlier findings [1] which have shown that an amplified RBS signal spreads during the linear stage of the instability and shrinks during the nonlinear stage.

The physical explanation of the nonlinear SRA is fairly intuitive. When $\omega_{B}$ exceeds $\omega_{p}$, all the terms in the RHS of Eq. (1) become much smaller than the ponderomotive term in the LHS. Hence, the particle motion is described by the nonlinear oscillator equation $\ddot{\psi}_{j}+\omega_{B}^{2} \sin \psi_{j}=0$. In the reference frame moving with the short pulse electrons enter the ponderomotive bucket with the initial "speed" $\dot{\psi}=-\Delta \omega$. If this speed is smaller than the bucket height $\dot{\psi}_{\max }=2 \omega_{B}$, electrons become trapped and execute a synchrotron oscillation in the bucket. For example, in the simulated case of $\Delta \omega=1.7 \omega_{B}$ electrons were initially entering the pulse near the maximum of the ponderomotive bucket. In a set of numerical simulations we varied the laser detuning between $-2.0 \omega_{p}<\Delta \omega<-1.5 \omega_{p}$ and did not see any qualitative differences in the SRA.

In the nonlinear regime signal duration adjusts itself, so that the trapped electrons make exactly half a bounce in the ponderomotive potential of the signal and the pump. Since the amount of energy transferred from pump to signal is proportional to the momentum deposited into the plasma electrons, $\pi / \omega_{B}$ pulse duration corresponds to the most efficient amplification of the pulse. As the signal
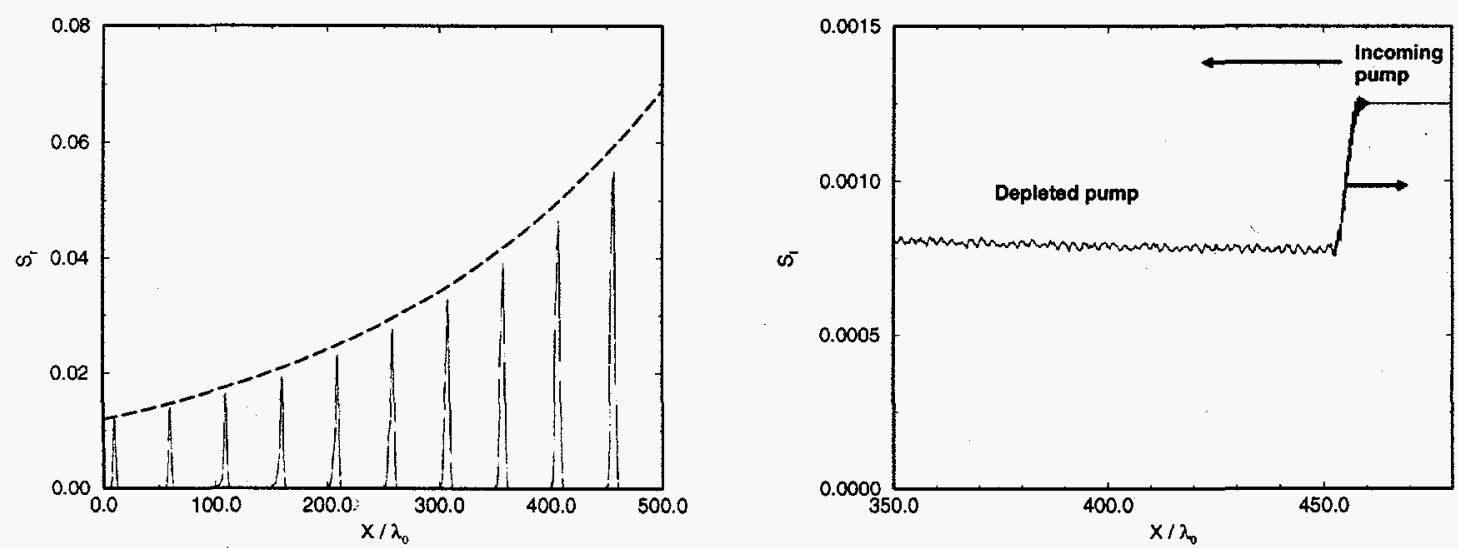

FIGURE 3. Superradiant amplification in the large pump depletion regime, results of the PIC simulation with 1-D VLPL code. Pump $a_{1}=0.025$, initial signal $a_{1}=0.07$ and $\tau_{L}=1 / \omega_{p}$, plasma $n_{0}=10^{19} \mathrm{~cm}-3$. 
is amplified, its duration decreases. In fact, a self-similar superradiant solution has been found by Bonifacio et. al. [7] in the context of free-electron lasers. The peak intensity of this solution increases quadratically (not exponentially!) with the propagation distance. For the RBS the growth of the superradiant spike was estimated earlier [1] as

$$
\left|a_{0}\right| \approx \frac{4 \omega_{p}}{3 \pi \omega_{0}} k_{p} z\left|a_{1}\right|
$$

Eq. (7) predicts that $\left|a_{0} / a_{1}\right|=10$ after $k_{p} z=500$. From Fig. 3 the ten-fold increase in amplitude occurs for $k_{p} z=700$, which is in qualitative agreement with the analytic estimate.

Pump depletion is very modest for tenuous plasmas. In the example shown in Fig. 3, with $\omega_{0} / \omega_{p}=20$, pump depletion is only $14 \%$. This translates into the low efficiency of short-pulse amplification. To demonstrate that much higher extraction efficiency is possible, we simulated a higher density case $\omega_{0} / \omega_{p}=10$ using the one-dimensional version of the VLPL PIC simulation code. As before, the pump amplitude is $a_{1}=0.025$, the initial signal intensity is $a_{0}=0.07$. The results are shown in Fig. 3. By the time the signal intensity grows by factor 5 pump depletion reaches $40 \%$.

Our quantitative understanding of the superradiant amplification is far from complete. However, the numerical and analytical results presented here indicate that both the efficient and manifold amplification of an ultra-short pulse is possible. It appears that SRA has the potential for becoming an efficient and simple pulsecompression technique.

\section{CONCLUSIONS}

In conclusion, we demonstrated analytically and numerically the electromagnetically-induced guiding of two counter-propagating lasers in the plasma at intensities much below the threshold for relativistic guiding. We also numerically demonstrated the possibility of superradiant amplification of ultra-short laser pulses by a properly detuned counter-propagating laser pump.

\section{ACKNOWLEDGMENTS}

The authors gratefully acknowledges helpful discussions with J. S. Wurtele. This work was supported by the DOE contract DE-FG030-98DP00210 and the United States Department of Energy (US DoE) Division of High Energy Physics.

\section{REFERENCES}

1. G. Shvets, J. S. Wurtele, and B. A. Shadwick, Phys. Plasmas, 4, 1872 (1997). 
2. A. Pukhov and J. Meyer-ter-Vehn, APS Bulletin 41, 1502 (1996).

3. E. Esarey et. al., Phys. Rev. Lett. 72, 2887 (1993).

4. G. Shvets and A. Pukhov, "Electromagnetically Induced Guiding of CounterPropagating Lasers in Plasmas", internal report PPPL-3297, May. 1998.

5. J. Durnin et. al., Phys. Rev. Lett. 58, 1499 (1987).

6. B. Hafizi, E. Esarey, and P. Sprangle, Phys. Rev. E 55, 3539 (1997).

7. R. Bonifacio, L. De Salvó, P. Pierini, N. Piovella, and C. Pellegrini, Phys. Rev. Lett. $\mathbf{7 3}, 70$ (1994), and references therein. 